\title{
The Effect of Power Density on Diffusion Length and Energy Gap of a-Si:H and nc-Si:H Thin Films Prepared by PECVD Technique
}

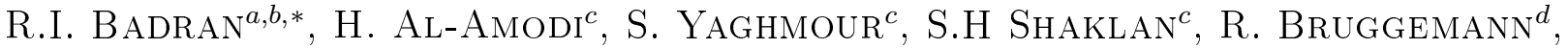 \\ X. $\mathrm{HAN}^{e}$ AND S. XIONG \\ ${ }^{a}$ Physics Department, The Hashemite University, P.O. Box 150459, Zarqa, Jordan \\ ${ }^{b}$ School of Natural Resources Engineering and Management, German-Jordanian University, Jordan \\ ${ }^{c}$ Physics Department, King Abdulaziz University, P.O. Box 80203, Jeddah, Saudi Arabia \\ ${ }^{d}$ Institut für Physik, Carl von Ossietzky Universität Oldenburg, 26111 Oldenburg, Germany \\ ${ }^{e}$ Institute of Optoelectronics, Nankai University, Tianjin, 300071, China
}

\begin{abstract}
The increase in power density of $0.3,0.5,0.6$, and $0.7 \mathrm{~W} \mathrm{~cm}^{-2}$ for hydrogenated amorphous and nanocrystalline silicon (a-Si:H and nc-Si:H) thin film samples prepared by plasma enhanced chemical vapor deposition technique causes an increase in crystalline volume fraction when the silane concentration is fixed. This increase in crystalline volume fraction is correlated to the absorption coefficient and refractive index which are determined from ellipsometric measurements. The crystallinity of samples is studied by both Raman and X-ray diffraction techniques. A mild change in the optical energy gap around an average value of $1.8 \mathrm{eV}$ is noticed due to the observed change in the degree of crystallinity of the samples when power density increases. Moreover, the ambipolar diffusion length measured by the steady-state photocarrier grating technique is found to change with the increase in power density. The values of some obtained optical parameters are compared to a standard crystalline sample.
\end{abstract}

PACS: 78.20.-e, 78.20.Ci, 73.61.Jc, 78.30.Ly

\section{Introduction}

Hydrogenated amorphous and nanocrystalline silicon (a-Si:H and nc-Si:H) have widespread applications in solar cells and transistors in addition to several other electronic devices. The importance of using both a-Si:H and nc-Si:H is due to ability of these materials to absorb light in different ranges of wavelengths, 300-700 nm and 500$900 \mathrm{~nm}$, respectively. This also allows the expansion of the range of light absorption in any possible fabricated a-Si/nc-Si tandem structured solar cell and may introduce a better efficiency. The study of the influence of different deposition parameters, using the plasma enhanced chemical vapor deposition (PECVD), on the growth of the material is important for understanding of the basic physics of the growth process of thin films and then on the fabrication of appropriate optoelectronic devices [1-7]. The ellipsometry, which is an easy and a nondestructive technique, provides information about several optical parameters like refractive index $n(h \nu)$, extinction

* corresponding author; e-mail: rbadran@hu.edu.jo, rbadran_i@yahoo.com coefficient $k(h \nu)$, absorption coefficient $\alpha(h \nu)$, film thickness, and optical energy gap. Several optical studies have been conducted using spectroscopic ellipsometry [8-10] on a-Si:H and nc-Si:H samples. The ellipsometric data were analyzed using the Tauc-Lorentz and Cody models.

In this paper, optical and transport properties of $\mathrm{Si}: \mathrm{H}$ thin film samples prepared by PECVD technique using $\mathrm{H}_{2}$-diluted $\mathrm{SiH}_{4}$ gases, at different power densities and a fixed silane concentration, $\mathrm{SC}=\mathrm{SiH}_{4} /\left(\mathrm{SiH}_{4}+\mathrm{H}_{2}\right)$, are studied. These properties are correlated to some structural aspects extracted from the Raman and X-ray diffraction (XRD) spectra. The Raman measurements have been conducted on the samples prepared at different power densities $\left(0.3,0.5,0.6\right.$, and $\left.0.7 \mathrm{~W} / \mathrm{cm}^{2}\right)$ when silane concentration $\mathrm{SC}=5.0 \%$, and the crystalline volume fraction for each sample is found. The ellipsometric data are collected at room temperature and analyzed for the samples using ellipsometry. Steady-state photocarrier grating (SSPG) technique is used to determine the ambipolar diffusion length. The effect of power density on crystalline volume fraction, diffusion length, optical energy gap, absorption coefficient, and refractive index has been discussed. 


\section{Experimental techniques}

\subsection{Sample preparation}

A set of amorphous and nanocrystalline silicon samples (S1-S4) has been prepared by PECVD technique, using $\mathrm{H}_{2}$-diluted $\mathrm{SiH}_{4}$ gases, at substrate temperature, pressure and silane concentration of $220^{\circ} \mathrm{C}, 1.6$ Torr, and $5 \%$, respectively, but at different values of power density 0.3 , $0.5,0.6$, and $0.7 \mathrm{~W} / \mathrm{cm}^{2}$. The measured values of thickness of samples S1, S2, S3 and S4 are 610, 450, 440, and $410 \mathrm{~nm}$ that correspond to deposition rates of 0.7 , $0.7,0.75,0.69 \mathrm{~W} / \mathrm{cm}^{2}$, respectively. All samples are deposited on Eagle 2000 Corning glass substrates.

\subsection{Raman and X-ray diffraction measurements}

The Raman scattering measurements were conducted by Renishaw RM2000, where a $514 \mathrm{~nm} \mathrm{Ar}{ }^{+}$excitation source is used. The crystallinity of hydrogenated nanocrystalline silicon (nc-Si:H) is extracted from the Raman spectra. The degree of crystallinity is estimated by finding the crystalline volume fraction $X_{\mathrm{c}}$.

XRD spectra are measured by Rigaku D/max 2500 , where $0.153 \mathrm{~nm} \mathrm{Cu} K_{\alpha}$ excitation source is used.

\subsection{Optical and transport measurements}

The spectroscopic ellipsometry (SE) measurements are conducted on a set of four samples using a variable angle spectroscopic ellipsometry (VASE) equipped with a goniometer. The data are collected when the measurements are carried out in air at room temperature in the wavelength range of 400-1600 $\mathrm{nm}$. Here, the SE measurements are obtained when the angles of incidence are 55,65 and 75 degrees in order to attain maximum reflected intensity from the sample and large oscillations in $\cos (\Delta)$ values within the measured wavelength range (where $\Delta$ is a well-known ellipsometric parameter that is related to the $p$ - and $s$-polarized light reflected from the sample). The refractive index and absorption coefficient, for hydrogenated amorphous or nanocrystalline silicon (a-Si:H or nc-Si:H) thin films are determined by $\mathrm{SE}$ in the measured wavelength range at room temperature using an optical Tau-Lorentz model. Moreover, the energy band gap of the sample is obtained.

A standard experimental setup of SSPG technique [11] is used to conduct measurements at room temperature. The SSPG measurements are carried out using a $\mathrm{He}-\mathrm{Ne}$ laser of the wavelength of $632.8 \mathrm{~nm}$ with a photon flux density of $10^{17} \mathrm{~cm}^{-2} \mathrm{~s}^{-1}$. The ratio of the main beam to small-signal beam intensities is chosen to be almost 5 in order to satisfy the requirement of the SSPG technique which implies that the ratio $\beta$ is independent of it. The voltage is chosen after checking that $\beta$ did not depend on it, thus insuring that the carrier distribution is governed by diffusion- and not by drift transport. The weak beam is chopped to a frequency of $30.8 \mathrm{~Hz}$.

Coplanar Al contacts were used for these electrical measurements where electrode distance is $12 \mathrm{~mm}$. Here, $\beta\left(=J_{\mathrm{coh}} / J_{\text {incoh }}\right)$ which is the ratio of the small signal current under coherent conditions with the presence of a grating, $J_{\text {coh }}$, to the small signal current under spatially homogeneous illumination $J_{\text {incoh }}$, has the formula

$$
\beta=1-2 \phi\left(1+\frac{4 \pi^{2} L_{\mathrm{amb}}}{\Lambda^{2}}\right)^{-2}
$$

that depends on the grating period $\Lambda[11,12]$. A well-known "Balberg plot of $\left(\frac{2}{1-\beta}\right)^{0.5}$ vs. $\Lambda^{-2}$ " which was deduced from the above formula, gives a straight line whose slope and intercept are equal to $\frac{\phi^{0.5}}{\left(2 \pi L_{\mathrm{amb}}\right)^{2}}$ and $-\frac{1}{\left(2 \pi L_{\mathrm{amb}}\right)^{2}}$, respectively [11].

The most important conditions that must be satisfied in the SSPG experiment are: (a) the applied voltage must be in the ohmic region of $I-V$ curve, (b) the value of $\beta$ must not change with increasing voltage, (c) a higher generation rate should be used in order to decrease errors in the experiment for films with a low-level signal, (d) surface scattering and surface recombination must be considered, especially when nc-Si:H sample is under study, for small grating periods $(\Lambda<2000 \mathrm{~nm})$ and for low illuminations.

The presence of surface scattering and recombination may cause some deviations from the straight line of the Balberg plot. The values of ambipolar diffusion length $L_{\text {amb }}$ and $\phi$ are obtained either from fitting the above-mentioned formula to the experimental data or from the Balberg plot. The parameter $\phi$ is related to the values of $\gamma$ (the exponent of the power-law dependence of photoconductivity versus generation rate), the dark conductivity reducing parameter, $\gamma_{\mathrm{d}}$, and the grating quality factor, $\gamma_{0}$, accounting for light scattering, mechanical vibrations, the low visibility of the fringes due to the nonideal polarization, etc. Here, the value of $\phi$ is clearly related to the quality of the surface of the films to optical scattering and has typical values between 0 and 1 $[11,12]$. The small value of $\phi$ may be an indication for the roughness of the film surface and this leads to an inaccurate measurement of diffusion length.

\section{Results and analysis}

Figure 1 shows the Raman spectra for four $\mathrm{Si}: \mathrm{H}$ thin films prepared at different power densities. The prominent peaks around $518 \mathrm{~cm}^{-1}$ may be indication for the presence of nanocrystallites in the silicon material. The mild shift in the peaks is an indication for the change in the degree of crystallinity with the change in power density. Here, the mild change in the deposition rate of the film around $0.7 \mathrm{~nm} / \mathrm{s}$ may have its effect on changing the spectrum shift in the Raman spectra from 517.6 to $519 \mathrm{~cm}^{-1}$. The Raman results show that the crystalline volume fraction $\left(X_{\mathrm{c}}=\left(I_{520}+I_{500}\right) /\left(I_{520}+I_{500}+I_{480}\right)\right.$, where $I_{500}, I_{520}$ and $I_{480}$ are the crystalline and amorphous intensity peaks which are extracted from the Raman scattering spectra at 500, 520, and $480 \mathrm{~cm}^{-1}$, respectively, using the Gaussian fitting curves shown in Fig. 1) increases with the increase in power density. 
TABLE

The crystalline volume fraction $X_{\mathrm{c}}$, ambipolar diffusion length $L_{\mathrm{D}}$, roughness, Urbach energy, $E_{\mathrm{U}}$ and optical energy gap $E_{\mathrm{g}}$ for $\mathrm{Si}: \mathrm{H}$ samples prepared at different power densities, with fixed substrate temperature, pressure and silane concentration $\left(\mathrm{SC}=\mathrm{SiH}_{4} /\left(\mathrm{SiH}_{4}+\mathrm{H}_{2}\right)\right)$ of $220^{\circ} \mathrm{C}, 1.6$ Torr and $5 \%$, respectively. The deposition rate for the samples changes mildly as $0.7,0.7,0.75,0.69 \mathrm{~W} / \mathrm{cm}^{2}$, due to power densities of 0.3 , $0.5,0.6$, and $0.7 \mathrm{~W} \mathrm{~cm}^{-2}$, respectively.

\begin{tabular}{c|c|c|c|c|c|c|c}
\hline \hline $\begin{array}{c}\text { Sample } \\
\text { No. }\end{array}$ & $\begin{array}{c}X_{\mathrm{c}} \\
{[\%]}\end{array}$ & $\begin{array}{c}\text { Power } \\
\text { density } \\
{\left[\mathrm{W} / \mathrm{cm}^{2}\right]}\end{array}$ & $\begin{array}{c}L_{\mathrm{amb}} \\
{[\mathrm{nm}]}\end{array}$ & $\phi$ & $\begin{array}{c}\text { Roughness } \\
{[\mathrm{nm}]}\end{array}$ & $\begin{array}{c}E_{\mathrm{g}} \\
{[\mathrm{eV}]}\end{array}$ & $\begin{array}{c}E_{\mathrm{U}} \\
{[\mathrm{meV}]}\end{array}$ \\
\hline $\mathrm{S} 1$ & 0 & 0.3 & $115 \pm 1.3$ & $0.072 \pm 0.010$ & $19.60 \pm 0.077$ & $1.761 \pm 0.139$ & $525 \pm 0.013$ \\
$\mathrm{~S} 2$ & 45 & 0.5 & $120 \pm 1.5$ & $0.142 \pm 0.018$ & $10.40 \pm 0.046$ & $1.785 \pm 0.041$ & $226 \pm 0.018$ \\
$\mathrm{~S} 3$ & 56 & 0.6 & $112 \pm 1.9$ & $0.009 \pm 0.006$ & $10.36 \pm 0.053$ & $1.747 \pm 0.049$ & $307 \pm 0.043$ \\
S4 & 62 & 0.7 & $111 \pm 1.0$ & $0.017 \pm 0.005$ & $8.89 \pm 0.026$ & $1.848 \pm 0.032$ & $158 \pm 0.005$
\end{tabular}

The values of $X_{\mathrm{c}}$ for the samples are listed in Table. XRD spectra (not shown) asserts the presence of nanocrystallites in the silicon material. The increase in power density is manifested through different characteristics of the developed materials, namely, (a) structural order of the amorphous network, consequently (b) the bonded hydrogen content and (c) formation of large strains in the films. The deposition induces some reduction in the disorder by satisfying dangling bonds and forming some strong $\mathrm{Si}-\mathrm{Si}$ bonds, etc., by the beginning of the increase in power density. This is because the growth precursors find some stable and higher energy sites, and then allow for some crystallization of the structurally disordered films.

The calculated dispersion curves of refractive index of all Si:H samples at different power densities are compared to the dispersion curve of crystalline silicon (c-Si) sample in Fig. 2. It is interesting to note that the nc-Si:H samples have dispersion curves close to the c-Si dispersion curve while the a-Si:H sample exhibits higher refractive indices at the whole range of photon energies.

Furthermore, the variation of absorption coefficient against the photon energy for prepared $\mathrm{Si}: \mathrm{H}$ samples, is shown in Fig. 3. The absorption spectra of samples are compared to the absorption spectrum of a c-Si sample where the samples with high crystallinity have absorption coefficients close to those of $\mathrm{c}-\mathrm{Si}$ while those with lower crystallinity show higher absorption, at photon energies larger than $2.0 \mathrm{eV}$. The optical absorption spectra with high crystallinity $\left(X_{\mathrm{c}}=62 \%\right)$ exhibit lower absorption than the amorphous sample at smaller energies $(<2.0 \mathrm{eV})$. However, Fig. 3 shows that the optical absorption for the a-Si:H sample, at larger energies $(>2.0 \mathrm{eV})$, is obviously higher than other that of nanocrystalline samples. Moreover, at these energies, the nanocrystalline samples at different power densities exhibit a mildly reduced absorption. This may be related to the ability of those samples of higher crystallinity to generate carriers at this range of energies [1].

The samples prepared at power density of the range 0.3-0.7 $\mathrm{W} \mathrm{cm}^{-2}$ exhibit a change in the Urbach energy, $E_{\mathrm{U}}$, between 158 and $525 \mathrm{meV}$, as seen in Table. This

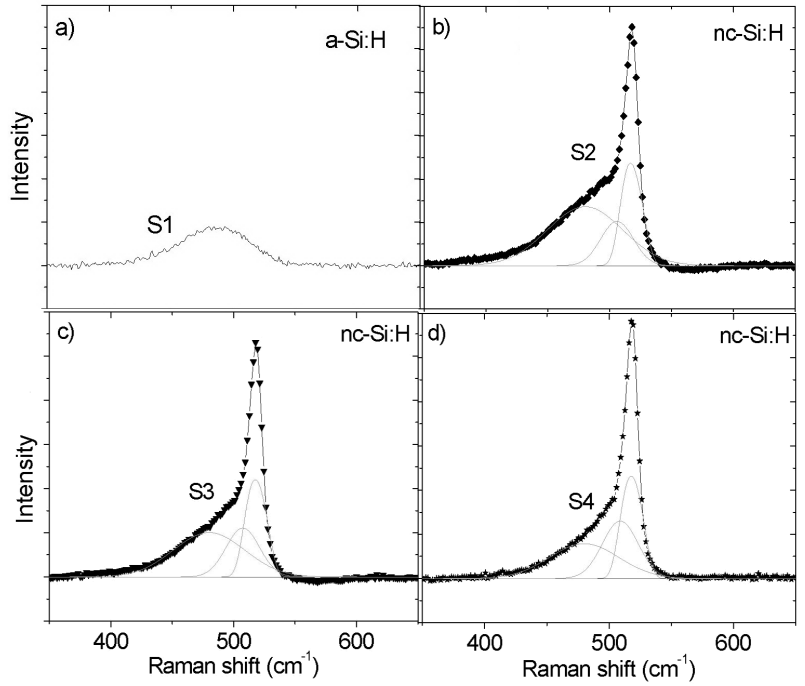

Fig. 1. Raman spectra for Si:H samples (S1-S4) prepared using PECVD technique with different power density values at substrate temperature, pressure and silane concentration density of $220^{\circ} \mathrm{C}, 1.6$ Torr and $\mathrm{SC}=5 \%$, respectively, are shown: (a) $X_{\mathrm{c}}=0$, (b) $X_{\mathrm{c}}=45 \%$, (c) $X_{\mathrm{c}}=56 \%$, and (d) $X_{\mathrm{c}}=62 \%$. The deconvolution Gaussian curves for the nc-Si:H samples (S2-S4) are also illustrated.

indicates that the exponential absorption tail, here, has a range wider than that of $250-300 \mathrm{meV}$ found by others [13]. In addition, this range is obviously wider than that 50-60 meV found for a-Si:H samples [5, 14, 15]. The increase in the crystalline volume fraction with increasing power density may cause simultaneous decrease in the grain size and increase the number of nanocrystalline grains. However, the decrease of the Urbach energy with increasing power density indicates the growth of defects and disorders which may be correlated, here, to the increase in the number of nanocrystalline grains and the accompanied grain boundaries.

Figure 4 shows the Balberg plots of our experimental data $[11,16]$. The least squares fit lines to the measured 


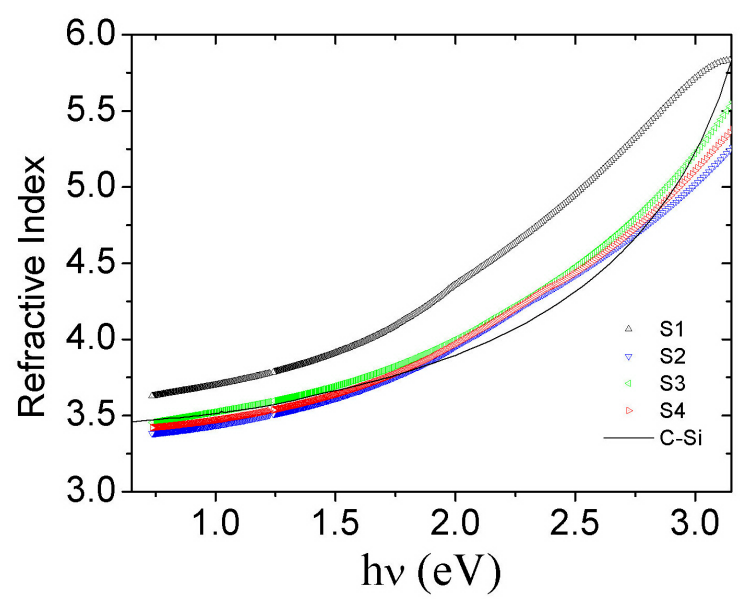

Fig. 2. Variation of refractive index, $n$, versus photon energy for $\mathrm{Si}: \mathrm{H}$ samples (S1-S4) prepared with different values of power density of (a) $0.3 \mathrm{~W} \mathrm{~cm}^{-2}$, (b) $0.5 \mathrm{~W} \mathrm{~cm}^{-2}$, (c) $0.6 \mathrm{~W} \mathrm{~cm}^{-2}$ and (d) $0.7 \mathrm{~W} \mathrm{~cm}^{-2}$ at substrate temperature, pressure and silane concentration of $220^{\circ} \mathrm{C}, 1.6$ Torr, and $5 \%$, respectively. The dispersion curve of refractive index of c-Si sample is also shown.

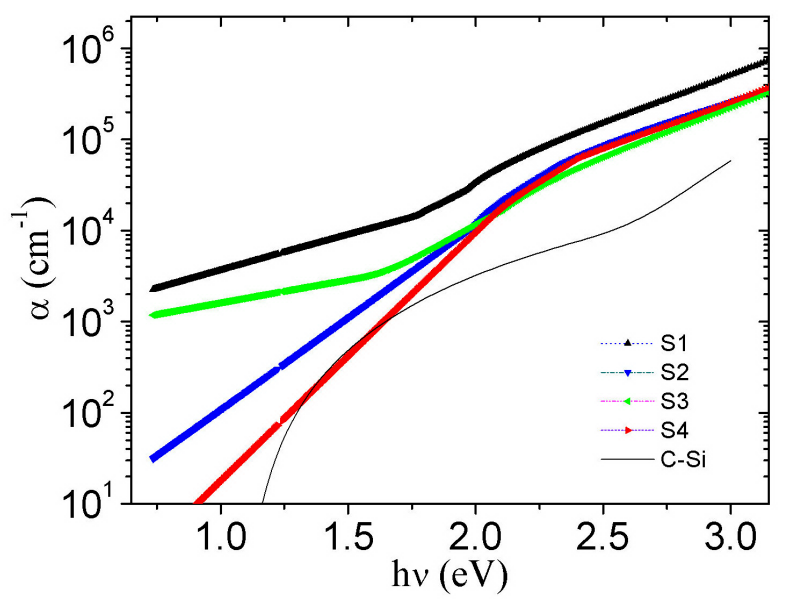

Fig. 3. Variation of absorption coefficient with photon energy for $\mathrm{Si}: \mathrm{H}$ samples (S1-S4) prepared with different values of power density of (a) $0.3 \mathrm{~W} \mathrm{~cm}^{-2}$, (b) $0.5 \mathrm{~W} \mathrm{~cm}^{-2}$, (c) $0.6 \mathrm{~W} \mathrm{~cm}^{-2}$, and (d) $0.7 \mathrm{~W} \mathrm{~cm}^{-2}$. The substrate temperature, pressure and silane concentration are $220^{\circ} \mathrm{C}, 1.6$ Torr, and $5 \%$, respectively. The absorption spectrum of a typical c-Si sample is compared to our absorption spectra of $\mathrm{Si}: \mathrm{H}$ samples.

$\left(\frac{2}{1-\beta}\right)^{0.5}$ vs. $\Lambda^{-2}$ are also shown. This allows for determining the values of $L_{\mathrm{amb}}$ and $\phi$ when the ambipolarity condition is fulfilled. The values of parameter $\phi=0.114$, 0.009 and 0.0172 for nanocrystalline films S2, S3, and S4, respectively, are an indication of the quality of optical scattering from surface patterns. Table shows that the change in the value of $\phi$ agrees to a certain extent with surface roughness obtained from ellipsometric analysis,

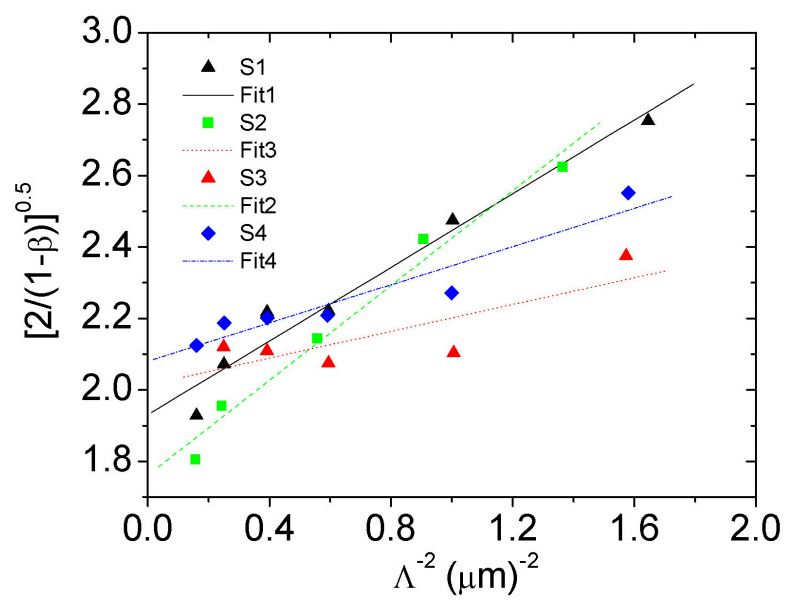

Fig. 4. Balberg plots of $\left(\frac{2}{1-\beta}\right)^{0.5}$ vs. $\Lambda^{-2}$ for Si:H samples S1, S2, S3 and S4 at four different power densities of $0.3,0.5,0.6$, and $0.7 \mathrm{~W} \mathrm{~cm}^{-2}$, respectively, and a fixed silane concentration $\mathrm{SC}=5 \%$.

apart from some anomalies which may be attributed to other factors like the optical scattering in the volume of the nc-Si:H film, $\gamma$ and $\gamma_{\mathrm{d}}$, etc. With larger film thickness the size of the crystallites increases, and consequently, surface roughness increases which leads to more light scattering [17]. Furthermore, $L_{\text {amb }}$ displays some dependence on sample thickness which is associated with the Fermi level pinning at the surface. Moreover, the increase in crystalline volume fraction of films due to the increase in power density leads to an increase of the number of nanocrystalline grains and accompanied grain boundaries which limit charge transport parallel to the substrate and consequently decreases $L_{\mathrm{amb}}$. However, the resistance to charge transport and consequently $L_{\mathrm{amb}}$ are found to exhibit different physical relations when grains touch each other from that when they are not in contact [18]. For the amorphous sample $\mathrm{S} 1$ (with $0.3 \mathrm{~W} \mathrm{~cm}^{-2}$ power density) which has relatively the largest thickness $(610 \mathrm{~nm})$ compared to that $(450,440$, and $410 \mathrm{~nm}$ ) of other samples, the optical scattering in the volume of nc-Si films may also contribute to total optical scattering and may lead to a smaller value of $\phi$ [17]. However, the small value of $\phi$ leads to an inaccurate measurement of diffusion length. Moreover, a mild change in energy gap (around an average of $1.79 \mathrm{eV}$ ) is shown due to the increase in power density in Table.

Most of the above results are consistent with the structural behaviour shown by the Raman spectra. However, sample $\mathrm{S} 3$ with power density of $0.6 \mathrm{~W} \mathrm{~cm}^{-2}$ has a strange behaviour in both the transport and optical properties. This may be attributed to a loose structure of Si:H, which has a lot of defects and disorder. The existence of larger defects and disorder may be due to the following reasons: (1) During the increase in power density (from 0.5 to $0.6 \mathrm{~W} / \mathrm{cm}^{2}$ ) with the mild increase in deposition rate (from 0.7 to $0.75 \mathrm{~nm} / \mathrm{s}$ for sample $\mathrm{S} 3$ ) a 
gradual reduction in the surface reaction rate may result due to the progressive changing of the available reaction time, in an attempt to retain similar sample thickness to that of the previous sample. As a result of that an enhancement of defect level which is created by credible number of unsaturated dangling bonds, becomes inevitable. (2) Or deposition system instability. Such increase in defects may allow for oxygen contamination in the sample post preparation. Here, oxygen plays the role of a donor, which causes a decrease in ambipolar diffusion length. The barrier of amorphous tissue between grain columns mainly limits the charge transport parallel to the substrate. Consequently, the grain column boundaries in addition to the oxygen contamination affect the ability of conduction for the nc-Si:H films [19]. In addition, the higher level of carriers generation, due to this power density, that enhances a reduced absorption at large energies can be understood. Here, this sample exhibits higher Urbach energy and lower energy gap than sample S2 with $X_{\mathrm{c}}=45 \%$.

\section{Conclusion}

The hydrogenated amorphous and nanocrystalline silicon samples prepared by PECVD under different power densities have been investigated. The optical parameters have been obtained from ellipsometric measurements. These results were found consistent with the structural behavior shown by the Raman and XRD spectra and also with those obtained by others. The increase in crystalline volume fraction is found correlated with the power density when silane concentration is kept fixed at $5 \%$. However, the increase in crystalline volume fraction from zero, 45,56 , to $62 \%$ leads to a corresponding change in diffusion length as 115, 120, 112 , and $111 \mathrm{~nm}$ and in the roughness from 19.6, 10.40, and 10.36 , to $8.9 \mathrm{~nm}$, respectively. Moreover, the energy gap changes mildly around an average value of $1.8 \mathrm{eV}$ when crystalline volume fraction changes from zero to $62 \%$. The behavior of our samples in visible and near infrared region of electromagnetic spectrum may allow them to be used in photovoltaic applications.

\section{References}

[1] Y.H. Wang, J. Lin, C.H.A. Huan, Mater. Sci. Eng. B 104, 80 (2003).

[2] Y. Chen, J. Wang, J. Lu, W. Zheng, J. Gu, S. Yang, X. Gao, Solar Energy 82, 1083 (2008).

[3] R.I. Badran, S. Al-Heniti, F.S. Al-Hazmi, A.A. Al-Ghamdi, J. Li, S. Xiong, J. Optoelectron. Adv. Mater. 11, 635 (2009).

[4] R.I. Badran, F.S. Al-Hazmi, S. Al-Heniti, A.A. Al-Ghamdi, J. Li, S. Xiong, Vacuum 83, 1023 (2009).

[5] M. Yamaguchi, K. Morigaki, Philos. Mag. B 79, 387 (1999).

[6] M. Yoon, K. Im, J. Yang, S. Lim, Physica B 405, 1526 (2010)

[7] Y. Bouizem, C. Abbes, J.D. Sib, D. Benlakehal, R. Baghdad, L. Chahed, K. Zellama, S. Charvet, J. Phys., Condens. Matter 20, 445221 (2008).

[8] R.W. Collins, A.H. Clark, S. Guha, C.-Y. Huang, J. Appl. Phys. 57, 4566 (1985).

[9] J.K. Saha, B. Bahardoust, K. Leong, A.B. Gougam, N.P. Kherani, S. Zukotynski, Thin Solid Films $\mathbf{5 1 9}$ 2863 (2011)

[10] D.K. Basa, G. Abbate, G. Ambrosone, U. Coscia, A. Marino, J. Appl. Phys. 107, 023502 (2010).

[11] D. Ritter, K. Weiser, E. Zeldov, J. Appl. Phys. 62, 4563 (1987); I. Balberg, K.A. Epstein, D. Ritter, Appl. Phys. Lett. 54, 2462 (1989).

[12] R.I. Badran, R. Bruggemann, R. Carius, J. Optoelectron. Adv. Mater. 11, 1464 (2009).

[13] P. Mishra, K.P. Jain, Mater. Sci. Eng. B 95, 202 (2002).

[14] Y. Kanemitsu, Phys. Rev. B 53, 13515 (1996).

[15] K. Murayama, S. Miyazaki, M. Hirose, Jpn. J. Appl. Phys. 31, L1358 (1992).

[16] R. Bruggemann, R.I. Badran, S. Xiong, J. Optoelectron. Adv. Mater. 9, 348 (2007).

[17] R. Bruggemann, P. Reing, M. Holling, Thin Solid Films 427, 358 (2003); R. Bruggemann, Appl. Phys. Lett. 73, 499 (1998).

[18] A. Fejfar, T. Mates, O. Certik, B. Rezek, J. Stuchlik, I. Pelant, J. Kocka, J. Non-Cryst. Solids 338-340, 303 (2004).

[19] J. Li, Y. Li, C. Wu, Z. Meng, S. Zhao, S. Xiong, Z. Lizhu, in: Proc. Asia Display, Vols. 1 and 2, East China Normal University, Shanghai 2007, p. 1178 